\title{
Universal Gap Fluctuations in the Superconductor Proximity Effect
}

\author{
M. G. Vavilov, P. W. Brouwer, and V. Ambegaokar \\ Laboratory of Atomic and Solid State Physics, Cornell University, Ithaca, New York 14853
}

\author{
C. W. J. Beenakker \\ Instituut-Lorentz, Universiteit Leiden, P.O. Box 9506, 2300 RA Leiden, The Netherlands
}

(Received 21 June 2000)

\begin{abstract}
Random-matrix theory is used to study the mesoscopic fluctuations of the excitation gap in a metal grain or quantum dot induced by the proximity to a superconductor. We propose that the probability distribution of the gap is a universal function in rescaled units. Our analytical prediction for the gap distribution agrees well with exact diagonalization of a model Hamiltonian.
\end{abstract}

DOI: $10.1103 /$ PhysRevLett.86.874

A normal metal in the proximity of a superconductor acquires characteristics that are typical of the superconducting state [1]. One of those characteristics is that the quasiparticle density of states vanishes at the Fermi energy. This superconductor proximity effect is most pronounced in a confined geometry, such as a thin metal film or metal grain, or a semiconductor quantum dot. In that case, provided the scattering in the normal metal is chaotic, no excitations exist within an energy gap $E_{g} \sim \hbar / \tau$, where $\tau$ is the typical time between collisions with the superconductor [2-7].

If the coupling to the superconductor is weak (as for the point contact coupling of Fig. 1), the functional form of the density of states becomes independent of microscopic properties of the normal metal, such as the shape, dimensionality, or mean free path. Weak coupling means that $\tau$ is much bigger than the time $\tau_{\text {erg }}$ needed for ergodic exploration of the phase space in the normal region [8]. For a point contact with $N \gg 1$ propagating modes at the Fermi level $\varepsilon=0$, the density of states has a square-root singularity at the excitation gap [4],

$$
\rho_{\mathrm{mf}}(\varepsilon)=\frac{1}{\pi} \sqrt{\frac{\varepsilon-E_{g}}{\Delta_{g}^{3}}} .
$$

For a ballistic point contact and in the absence of a magnetic field, $E_{g}=c N \delta$ is the mean-field energy gap and $\Delta_{g}=c^{\prime} N^{1 / 3} \delta$, where $c=0.048$ and $c^{\prime}=0.068$ are numerical constants and $\delta$ is the mean level spacing in the normal metal when it is decoupled from the superconductor.

Equation (1) was obtained in a self-consistent diagrammatic perturbation theory that uses $\tau \delta / \hbar \sim N^{-1}$ as a small parameter. Such a mean-field theory provides a smoothed density of states for which energies can be resolved only on the scale of the rate $\hbar / \tau \sim N \delta$ between collisions with the superconductor, not on smaller energy scales, and is unable to deal with mesoscopic sample-tosample fluctuations of the excitation gap. Mesoscopic fluctuations arise, e.g., upon varying the shape of a quantum dot or the impurity configuration in a metal grain. The lowest excited state $\varepsilon_{1}$ fluctuates from sample to sample
PACS numbers: 73.23. $-b, 74.50 .+r, 74.80 . F p$

around the mean-field value $E_{g}$, with a probability distribution $P\left(\varepsilon_{1}\right)$. It is the purpose of this paper to go beyond mean-field theory and to study the mesoscopic fluctuations of the excitation spectrum close to $E_{g}$. Our main result is that the gap distribution $P\left(\varepsilon_{1}\right)$ is a universal function of the rescaled energy $x=\left(\varepsilon_{1}-E_{g}\right) / \Delta_{g}$, in a broad range $|x| \ll N^{2 / 3}$, where $\Delta_{g}$ is defined in terms of the mean-field density of states (1). The Fermi level itself $(\varepsilon=0)$ falls outside this range, which is why the universal gap distribution was not found in a recent related study [9]. Our main findings are illustrated in Fig. 2. Note that the width of the gap distribution $\Delta_{g} \sim E_{g}^{1 / 3} \delta^{2 / 3}$ is parametrically smaller than the gap size $E_{g}$ but bigger than the mean level spacing $\delta$ in the dot.

Also note that, in terms of the rescaled variable $x$, the mean-field density $\rho_{\mathrm{mf}}$ is already universal, $\rho_{\mathrm{mf}}(x)=$ $\pi^{-1} x^{1 / 2}$, though $\rho_{\mathrm{mf}}$ is different from the true ensemble averaged density of states $\langle\rho\rangle$; see Fig. 2 . The difference could arise, because the mean-field theory is unable to resolve the density of states on the energy scale $\Delta_{g}$.

We first consider the gap distribution in the absence of a magnetic field and then include a time-reversal symmetry

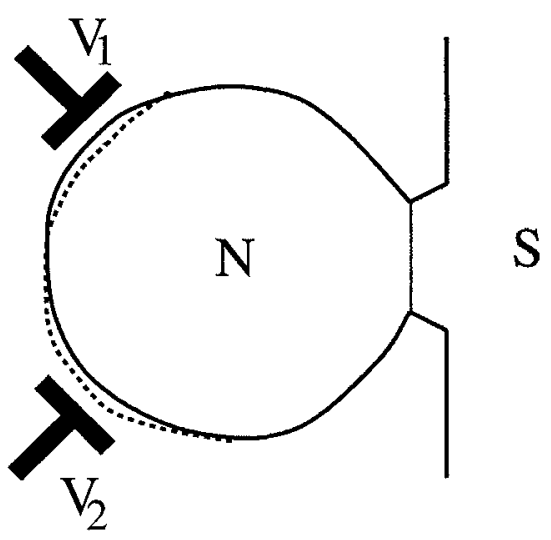

FIG. 1. A quantum dot (N) connected to a superconductor (S). The voltages on the gates $V_{1}$ and $V_{2}$ change the shape of the dot. Different values of the applied voltages create different samples within the same ensemble. 


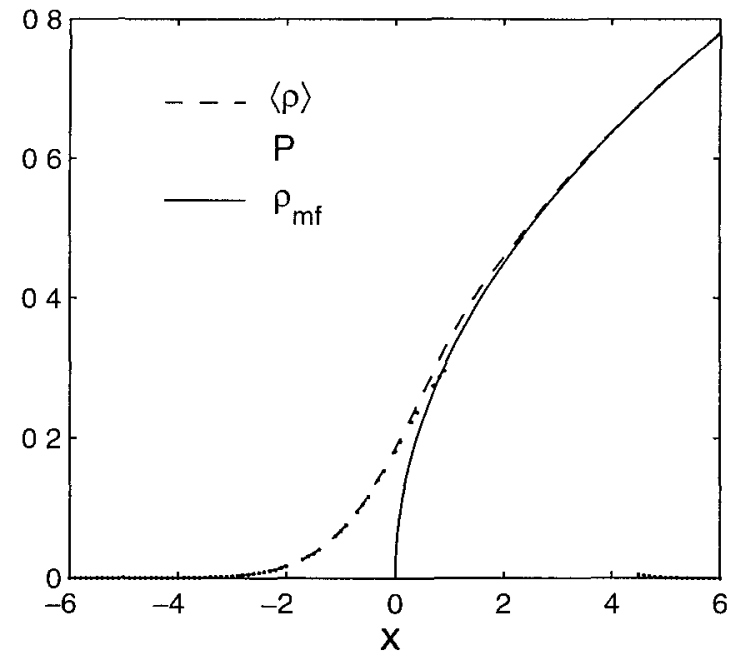

FIG 2 Mean-field and ensemble averaged density of states $\rho_{\mathrm{mf}}$ and $\langle\rho\rangle$, together with the probability distribution $P$ of the excitation gap, as a function of the rescaled energy $x=$ $\left(\varepsilon_{1}-E_{g}\right) / \Delta_{g}$ These curves are the universal predictions of the random-matrix theory

bıeakıng magnetic field The startıng point of our calculation is the effective Hamiltonian for a quantum dot coupled to a superconductor $[10]$,

$$
\mathcal{H}=\left(\begin{array}{cc}
H & -\pi W W^{\dagger} \\
-\pi W W^{\dagger} & -H^{*}
\end{array}\right)
$$

Here $H$ is an $M \times M$ Hermitian matrix representing the Hamiltonian of the isolated quantum dot, and $W$ is an $M \times N$ matrix that describes the coupling to the superconductor via an $N$-mode point contact For a ballistic point contact, $W_{m n}=\pi^{-1} \delta_{m n}(M \delta)^{1 / 2}$ [11] The number $M$ is sent to infinity at the end of the calculation [12] The effective Hamiltonian is a valid description of the low-lying excitations if the 1ate $N \delta$ of collisions with the superconductor ( $1 \mathrm{e}$, the escape rate from the normal quantum dot) is much smalle1 than the oider parameter $\Delta$ of the bulk superconductor See Ref [10] for a microscopic derivation of Eq (2) In the absence of a magnetic field, the matrix $H$ is symmeti1c To describe an ensemble of chaotic quantum dots (or disordered metal grains), we take $H$ from the Gaussian orthogonal ensemble (GOE) of random-matrix theory [13],

$$
\mathcal{P}(H) \propto \exp \left(-\frac{\pi^{2}}{4 \delta^{2} M} \operatorname{Tr} H^{2}\right)
$$

The choice of the distribution (3) is justified, since both characteistic energy scales $E_{g}$ and $\Delta_{g}$ of our problem are small compared to the inverse ergodic time $\hbar / \tau_{\text {erg }}$ (This is the Thouless energy of the isolated quantum dot ) In this case, valıdity of random-matrix theory for the Hamitonian $H$ of the isolated quantum dot is known to be valid for dots with diffusive [14] and ballistic chaotic [15] electron dynamics
Calculation of the density of states of $\mathcal{H}$ using peitur bation theory in $N^{-1}$ yields the result (1) discussed in the intioduction Our problem is to go beyond peitubation theory and find the probability distribution $P\left(\varepsilon_{1}\right)$ of the lowest positive eigenvalue $\varepsilon_{1}$ of the Hamiltonian (2)

We have solved this problem numetically by exact diagonalızation of the effectıve Hamitonian $\mathcal{H}$ Before presentıng these results, we first describe an entinely different approach, which leads to an analytical piediction for the gap distribution We invoke the universality hypothesis of random-matrix theory, that the local spectial statistics of a chaotic system depends only on the symmetiy prope1ties of the Hamiltonian, and not on microscopic properties This universality hypothesis has been proven for a broad class of Hamiltonians in the bulk of the spectium [16] but is believed to be valid near the edge of the spectrum as well A proof exists for so-called trace ensembles, having $\mathcal{P}(H) \propto \exp [-\operatorname{tr} f(H)]$, with $f$ an arbitrary polynomial function [17]

The mean-field density of states near the edge can be written in the form

$$
\rho_{\mathrm{mf}}(\varepsilon)=\frac{1}{a}\left(\frac{\varepsilon-b}{a}\right)^{p}, \quad \varepsilon>b
$$

According to the universality hypothesis, the spectral statistics neat the edge, in rescaled variables $(\varepsilon-b) / a$, depends only on the exponent $p$ and on the symmetry index $\beta[\beta=1$ (2) in the piesence (absence) of time-reversal symmetry] Generically, $p$ is either $1 / 2$ (soft edge) or $-1 / 2$ (hard edge) For our problem, we have $\beta=1, p=1 / 2, a=\pi^{2 / 3} \Delta_{g}, b=E_{g}$, cf Eq (1) The coriesponding gap distribution is given by [18]

$$
\begin{gathered}
P(\varepsilon)=\frac{d}{d \varepsilon} F_{1}\left[\left(\varepsilon-E_{g}\right) / \Delta_{g}\right] \\
F_{1}(x)=\exp \left(-\frac{1}{2} \int_{-\infty}^{x}\left[q\left(x^{\prime}\right)+\left(x-x^{\prime}\right) q^{2}\left(x^{\prime}\right)\right] d x^{\prime}\right)
\end{gathered}
$$

The function $q(x)$ is the solution of

$$
q^{\prime \prime}(x)=-x q(x)+2 q^{3}(x),
$$

with asymptotic behavior $q(x) \rightarrow \operatorname{Al}(-x)$ as $x \rightarrow-\infty$ [ $\mathrm{Al}(x)$ being the Airy function]

The distribution (5) is shown in Fig 3 (solid curve) It is centered at a positive value of $x=\left(\varepsilon_{1}-E_{g}\right) / \Delta_{g}$, meaning that the average gapsize $\left\langle\varepsilon_{1}\right\rangle_{1}$ is about $\Delta_{g}$ bigger than the mean-field gap $E_{g}$ For small $x$ there is a tarl of the form

$$
P(x) \approx \frac{1}{4 \sqrt{\pi|x|^{1 / 4}}} \exp \left(-\frac{2}{3}|x|^{3 / 2}\right), \quad x \ll-1
$$

Nonuniveisal corrections to the distribution (5) become important for energy differences $\left|\varepsilon-E_{g}\right| \geqslant E_{g}$, hence for $|x| \geqslant N^{2 / 3} \quad$ Since the width of the gap distribution 


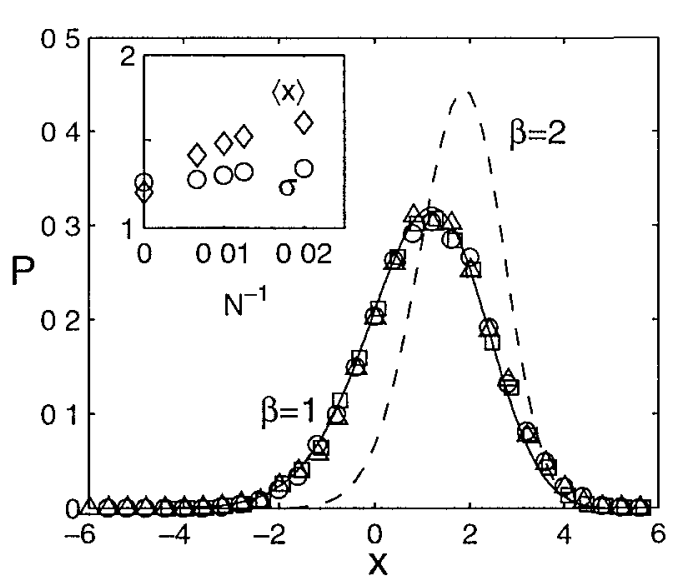

FIG 3 Probability distribution of the rescaled excitation gap $x=\left(\varepsilon_{1}-E_{g}\right) / \Delta_{g}$ Data points follow from an exact diagonalization of $10^{4}$ realizations of the effective Hamiltonian (2) for different values of $M$ and $N(\triangle M=400, N=200$, $\square \quad M=600, N=150, \quad \bigcirc \quad M=600, N=80$ ) The solid curve is the universal prediction (5) of randommatrix theory The mean of the data points has been adjusted to fit the curve by applying a horizontal offset, no other fit parameters are involved The inset shows the actual mean $\langle x\rangle$ and root-mean-square value $\sigma$ of the data for $M / N=4$ for different values of $N$, together with the random-matrix prediction for $N \rightarrow \infty$ These 1esults are all in zero magnetic field The dashed curve is the random-matrix theory prediction (15) in the presence of a time-reversal symmetry breaking magnetic field $(\beta=2)$

is of order unity in the variable $x$, the probability to find a sample with an excitation gap in the nonuniversal regime is exponentially small

In order to verify our univeisality hypothesis, we compaie Eq (5) with the results of an exact diagonalization of the Hamiltonian (2) As one can see in Fig 3, the numerical data are in good agieement with the analytical prediction The small deviations can be attıbuted to the finiteness of $N$ and $M$ in the numeics

Let us now consider the effect of a weak magnetic field on the gap distribution In the effective Hamitonian, the presence of a magnetic field is described by replacing $H$ by [19]

$$
H(\alpha)=H+\imath \alpha A
$$

Here $A$ is an $M \times M$ real antisymmetric matrix, whose off-diagonal elements have the same variance as those of $H$ The parameter $\alpha$ is propoitional to the magnetic field,

$$
M \alpha^{2}=\eta\left(\frac{\Phi}{\Phi_{0}}\right)^{2} \frac{\hbar}{\tau_{\mathrm{erg}} \delta}
$$

where $\Phi$ is the magnetic flux through the quantum dot, $\Phi_{0}=h / e$ is the flux quantum, and $\eta$ is a nonuniversal numerical constant [11] The case $\alpha=0$ conesponds to the GOE that we considered above, the case $\alpha=1$ corresponds to the Gaussian unitaly ensemble (GUE) of fully bioken time reveisal symmetry
The effect of a magnetic field on the density of states in mean field theory is known [4] The square-1oot singulanty (1) near the gap strll holds, but the magnitude of the gap is reduced The critical flux $\Phi_{c}$ at which $E_{q}=0$ and hence the proximity effect is fully suppressed is given by

$$
M \alpha^{2} \sim N \Rightarrow \Phi_{c} \sim \Phi_{0} \sqrt{\frac{N \tau_{\mathrm{erg}} \delta}{\hbar}}
$$

This is a much large flux than the flux $\Phi_{\text {bulk }}$ at which the spectral statistics in the bulk of the spectrum ciosses over from GOE to GUE, which is given by [19]

$$
M \alpha^{2} \sim 1 \Rightarrow \Phi_{\text {bulk }} \sim \Phi_{0} \sqrt{\frac{\tau_{\text {erg }} \delta}{\hbar}}
$$

We now argue that the characteristic flux $\Phi_{\text {edge }}$ for the spectial statistics at the edge of the spectrum is intermedi ate between $\Phi_{c}$ and $\Phi_{\text {bulk }}$ We consider the effect of the magnetic field on the lowest eigenvalue $\varepsilon_{1}$ of $\mathcal{H}$ to second order in perturbation theory,

$$
\delta \varepsilon_{1}=\sum_{j \neq 1} \alpha^{2} \frac{|\langle 1|\mathcal{A}| J\rangle|^{2}}{\varepsilon_{1}-\varepsilon_{J}}, \quad \mathcal{A}=\iota\left(\begin{array}{cc}
A & 0 \\
0 & -A
\end{array}\right)
$$

Since typically $|\langle 1|\mathcal{A}| 2\rangle|^{2} \sim M \delta^{2} / \pi^{2}$ and $\varepsilon_{2}-\varepsilon_{1} \sim$ $\Delta_{g}$, we see that the effect of level repulsion from the neighboing level $\varepsilon_{2}$ on the lowest level $\varepsilon_{1}$ becomes comparable to $\Delta_{g} \sim N^{1 / 3} \delta$ if

$$
M \alpha^{2} \sim N^{2 / 3} \Rightarrow \Phi_{\text {edge }} \sim \Phi_{0} \sqrt{\frac{N^{2 / 3} \tau_{\text {erg }} \delta}{\hbar}}
$$

The terms in Eq (13) with $J \gg 1$ give a uniform shift of all low-lying levels and, hence, do not affect the fluc tuations Fol $N \gg 1$ the flux scale (14) for breaking tume-reversal symmetry at the edge of the spectrum is much smallel than the critical flux $\Phi_{c}$ needed to suppiess the proximity effect Indeed, using $N \sim E_{g} / \delta$ we find $\Phi_{\text {edge }} \sim \Phi_{0} \tau_{\text {erg }}^{1 / 2} E_{g}^{1 / 3} \delta^{1 / 6}$, which is much smaller than $\Phi_{c} \sim \Phi_{0} \tau_{\operatorname{erg}}^{1 / 2} E_{g}^{1 / 2}$ Notice that the naive substitution of $\delta$ by $\Delta_{g}$ in expression (12) for $\Phi_{\text {bulk }}$ would give the wrong result for $\Phi_{\text {edge }}$

To study numerically the ciossover in the gap fluctuation statistics, $N^{2 / 3} \ll N$ has to be satisfied, which is difficult The analytical prediction for fully broken time-ieversal symmetry is [18]

$$
\begin{gathered}
P(\varepsilon)=\frac{d}{d \varepsilon} F_{2}\left[\left(\varepsilon-E_{g}\right) / \Delta_{g}\right] \\
F_{2}(x)=\exp \left(-\int_{-\infty}^{x}\left(x-x^{\prime}\right) q^{2}\left(x^{\prime}\right) d x^{\prime}\right)
\end{gathered}
$$

This curve is shown dashed in Fig 3 The tail for small $x$ is now given by

$$
P(x) \approx \frac{1}{8 \pi|x|} \exp \left(-\frac{4}{3}|x|^{3 / 2}\right), \quad x \ll-1
$$

The gap fluctuations are significantly ieduced by 
TABLE I Characteristıc energy and magnetic flux scales for the spectral statistics in the bulk and at the edge of the spectrum and for the size of the gap

\begin{tabular}{lcc}
\hline \hline & Energy scale & Flux scale \\
\hline Bulk statıstics & $\delta$ & $\Phi_{0} \tau_{\mathrm{crg}}^{1 / 2} \delta^{1 / 2} / \hbar^{1 / 2}$ \\
Edge statıstıcs & $E_{\delta}^{1 / 3} \delta^{2 / 3}$ & $\Phi_{0} \tau_{\mathrm{crg}}^{1 / 2} \delta^{1 / 6} E_{\delta}^{1 / 3} / \hbar^{1 / 2}$ \\
Gap size & $E_{\delta}$ & $\Phi_{0} \tau_{\mathrm{crg}}^{1 / 2} E_{g}^{1 / 2} / \hbar^{1 / 2}$ \\
\hline \hline
\end{tabular}

application of a magnetic field, see Fig 3 and Eqs (8) and (17) The suppression of the fluctuations is a generic feature of the different level statistics for ensembles with orthogonal and unitary symmetries, the ensembles with less symmetry (the unitary ensemble) having a more rigid and, hence, less fluctuating spectrum [13]

To make contact with Ref [9] we briefly discuss the $1 \mathrm{~m}$ plications of our tesults for the ensemble averaged density of states $\langle\rho(\varepsilon)\rangle$ in the subgap regime The tall of $P(x)$ for $x \leqslant-1$ is the same as the tarl of $\langle\rho\rangle$, cf Fig 2 We conclude that $[20]$

$$
\langle\rho(x)\rangle \propto \exp \left(-\frac{2 \beta}{3} x^{3 / 2}\right)
$$

over a broad range $\Delta_{g} \ll E_{g}-\varepsilon \ll E_{g}$ inside the meanfield gap A different exponential decay (with a power of 2 instead of $3 / 2$ in the exponent) was predicted iecently by Beloborodov, Narozhny, and Aleine1 [9], for the subgap density of states of an ensemble of superconducting grains in a weak magnetic field Since the mean-field density of states in that problem is also of the form (1), the universal GUE edge statistics should apply The reason that the un1versal decay (18) was not obtaned in Ref [9] is that then theory applies to the nonuniversal energy range $\varepsilon \ll E_{g}$ near the Fermı level To emphasize the significance of the universal energy iange we note that the probability to have the lowest energy level in that range is larger than in the nonuniversal range by an exponentrally large factor $\propto \exp \left[\left(E_{g} / \Delta_{g}\right)^{3 / 2}\right]$

In conclusion, we have argued that the proximity effect in a mesoscopic system has a gap distribution which is universal once energy is measured in units of the energy scale $\Delta_{g} \propto\left(E_{g} \delta^{2}\right)^{1 / 3}$ defined from the mean-field density of states $\rho(\varepsilon)=\left[\left(\varepsilon-E_{g}\right) / \Delta_{g}^{3}\right]^{1 / 2} / \pi \quad$ This universal distıbution is the same as the distribution of the smallest eigenvalue of the Gaussian orthogonal or unitary ensembles from random-matrix theory, depending on whether time-reversal symmetry is broken or not We have identıfied the magnetic field scale for breaking tıme-reversal symmetry and verified our results by exact diagonalızation of an effectıve Hamiltonian Charactersstic energy and magnetic field scales are summarized in Table I The universality of our prediction should offer ample opportunities for experimental observation
We thank I Aleınet, I Beloborodov, E Mishchenko, and B Narozhny for useful discussions This work was supported by the Cornell Center for Materials Research under NSF Grant No DMR-9632275 and by the Dutch Science Foundation NWO/FOM

[1] M Tinkham, Introduction to Superconductivity ( $\mathrm{McG}_{1}$ awHill, New York, 1995)

[2] A A Golubov and M Yu Kupriyanov, Sov Phys JETP 69, 805 (1989)

[3] W Belzig, C Bruder, and G Schon, Phys Rev B 54, 9443 (1996)

[4] J A Melsen, PW Brouwer, KM Frahm, and C W J Beenakker, Europhys Lett 35, 7 (1997), Phys Scrip 69, 223 (1997)

[5] A Lodder and Yu V Nazarov, Phys Rev B 58, 5783 (1998)

[6] F Zhou, P Charlat, B Spivak, and B Pannetier, J Low Temp Phys 110, 841 (1998)

[7] W Ihra, M Leadbeater, J L Vega, and K Richter condmat/9909100

[8] In a quantum dot or metal grain of size $R$, with Fermı velocity $v_{\mathrm{F}}$ and mean free path $\ell$, one has $1 / \tau_{\mathrm{erg}} \sim$ $v_{\mathrm{F}} R^{2} \min (\ell, R)$

[9] I S Beloborodov, B N Narozhny, and I L Aleuner, Phys Rev Lett 85, 816 (2000)

[10] K M Frahm, PW Brouwer, J A Melsen, and C W J Beenakker, Phys Rev Lett 76, 2981 (1996)

[11] C W J Beenakker, Rev Mod Phys 69, 731 (1997)

[12] In our numerical computations it was necessary to choose the ratio $M / N$ relatively small in order to achieve the limit $N \gg 1$ needed for universality of the gap distribution For finite $M / N$, the mean-field result (1) still holds, but now with coefficients $c$ and $c^{\prime}$ for the energy scales $E_{g}$ and $\Delta_{g}$ that weakly depend on $M / N$ For the comparison of the numerical data with the prediction of random-matrix theory we calculated $E_{g}$ and $\Delta_{\delta}$ from the mean-field theory [Eq (8) of Ref [4]] for the values of $M / N$ used in the exact diagonalizations No fit parameters are involved in this procedure

[13] M L Mehta, Random Matrices (Academic, New York, 1991)

[14] K B Efetov, Supersymmetry in Disorder and Chaos (Cambridge University Press, Cambridge, England, 1996)

[15] A V Andreev, O Agam, B D Simons, and B L Altshuler, Phys Rev Lett 76, 3947 (1996)

[16] T Guhr, A Muller-Groeling, and H A Weidenmuller, Phys Rep 299, 189 (1998)

[17] E Kanzieper and V Fresllkher, Phys Rev Lett 78, 3806 (1997)

[18] C A Tracy and H Widom, Commun Math Phys 159, 151 (1994), 177, 727 (1996)

[19] A Pandey and M L Mehta, Commun Math Phys 87, 449 (1983)

[20] The complete random matrix-theory piediction is $\langle\rho(x)\rangle=$ $-x \operatorname{Ar}^{2}(x)+\left[\mathrm{Al}^{\prime}(x)\right]^{2}+\frac{1}{2} \delta_{\beta 1} \mathrm{Al}_{1}(x)\left[1-\int_{\lambda}^{\infty} \mathrm{Al}_{1}(y) d y\right]$ The $\beta=1$ result is plotted in Fig 2 